\title{
A Case of Auditory Neuropathy Caused by Pontine Hemorrhage in an Adult
}

\author{
Seung-Hyun Chung, Sung Wook Jeong, and Lee-Suk Kim \\ Department of Otolaryngology-Head and Neck Surgery, Dong-A University College of Medicine, Busan, Korea
}

Received November 11,2016

Revised December 20, 2016

Accepted December 27, 2016

Address for correspondence

Sung Wook Jeong, MD, PhD

Department of Otolaryngology-

Head and Neck Surgery,

Dong-A University

College of Medicine,

26 Daesingongwon-ro,

Seo-gu, Busan 49201, Korea

Tel +82-51-240-5428

Fax +82-51-253-0712

E-mail dau_su@naver.com
A pontine hemorrhage can evoke several neurological symptoms because the pons contains various nuclei and nerve fibers. Hearing loss can develop as a result of a pontine hemorrhage because there is an auditory conduction pathway in the cochlear nucleus of the pons. However, very few cases of hearing loss caused by pontine lesions have been reported, and there have been no reports of auditory neuropathy that developed following a pontine hemorrhage. Recently we had a patient who experienced a nontraumatic pontine hemorrhage who was diagnosed with auditory neuropathy. The 34-year-old male patient was admitted to the emergency department with sudden alteration of mental status. His brain computed tomographic imaging revealed a hemorrhage in the central pons. He complained of hearing difficulties after his mental status recovered through conservative treatment, but a pure-tone audiogram showed very mild hearing loss in both ears. Further hearing tests using otoacoustic emissions, which showed normal responses, and auditory brainstem responses, which showed no waveforms at maximum stimulus intensity, revealed that his hearing difficulties were caused by auditory neuropathy. This case implies that the threshold of sound detection can be preserved in patients with pontine hemorrhage who complain of hearing difficulties. Auditory neuropathy should be considered as a possible cause of hearing difficulties in these patients and appropriate hearing tests should be performed.

J Audiol Otol 2017;21(2):107-111

KEY WORDS: Auditory neuropathy · Pontine hemorrhage $\cdot$ Central hearing loss.

\section{Introduction}

Nontraumatic intracranial hemorrhage can be caused by hypertension, vasculitis, aneurysm, or coagulopathy, and mainly occurs in the brain parenchyma, subarachnoid space, and subdural space, with the brain parenchyma the most common site of the lesion. Pontine hemorrhage accounts for about $10 \%$ of nontraumatic intracranial hemorrhage [1]. Because there are many neural structures in the pons, including the abducens nucleus, trigeminal nucleus, cochlear nucleus, and vestibular nucleus, a pontine hemorrhage can cause a variety of neurological symptoms depending on the amount of bleeding and the location in which bleeding occurs [2]. The initial clinical signs of pontine hemorrhage may vary, ranging from mild confusion

This is an Open Access article distributed under the terms of the Creative Commons Attribution Non-Commercial License (http://creativecommons.org/licenses/by-nc/4.0/) which permits unrestricted non-commercial use, distribution, and reproduction in any medium, provided the original work is properly cited. to coma. Half of the patients show miosis at the initial clinical examination, but some can have normal pupils or mydriasis. Most patients have normal body temperature, while clinical symptoms such as respiratory disturbance, facial nerve palsy, abducens nerve palsy, vagal nerve palsy, hearing loss, and disequilibrium may occur. Tetraplegia is the most common motor disorder, but hemiparesis or normal motor function can be present [3]. The treatment of a pontine hemorrhage is limited to conservative care and surgery is not indicated in most cases. The prognosis is very poor and the mortality rate is reported to be $40-60 \%$ [4].

If a hemorrhagic lesion develops in an auditory conduction pathway involving the cochlear nucleus in the pons, sensorineural hearing loss can occur [5]. Here, we report a patient with a pontine hemorrhage who developed hearing difficulties as a result of auditory neuropathy. To our knowledge, this is the first report showing that a pontine hemorrhage can cause hearing loss as a form of auditory neuropathy. 


\section{Case Report}

A 34-year-old man was admitted to the emergency room with sudden mental deterioration, dysphonia, and limb weakness. He had no relevant past medical history such as hypertension, chronic hyperinsulinemia, or diabetes. His blood pressure, light reflex, and blood tests related to coagulopathy were normal. His level of consciousness was drowsy at initial presentation, but subsequently worsened to stupor. He showed sudden respiratory difficulty, so underwent endotracheal intubation. Because a stroke was suspected, a brain computed tomography (CT) was performed. The CT scan revealed a hemorrhagic lesion in the central pontine area. The amount of bleeding was estimated to be approximately $5 \mathrm{~mL}$ (Fig. 1). He was admitted to the intensive care unit (ICU) and received conservative treatment because surgical intervention was impossible. Hemostatics, antihypertensive agents, and anticonvulsants were administered. The hemorrhagic lesion in the pons was reduced in size on subsequent brain CT scans, and his mental status also improved. His mental status was restored to the alert level and self-respiration was possible without mechanical ventilation 7 days after ICU admission. After he recovered his mental status, he complained of hearing difficulty. He said that he could hear speech sounds, but could not understand them at all. Physical examination of both ears revealed normal tympanic membranes. His average hearing threshold by pure tone audiometry was $26 \mathrm{~dB} \mathrm{HL}$ in the right ear and $32 \mathrm{~dB}$ HL in the left ear (Fig. 2), but his speech discrimination score was zero for both ears. Further hearing tests were performed to reveal the cause of hearing loss. Transiently evoked otoacoustic emission (OAE) and distortion product OAE showed robust responses for both ears, but the auditory brainstem response (ABR) showed only a broad wave I at 100 $\mathrm{dB}$ nHL click stimuli (Figs. 3, 4). Based on these results, he was diagnosed as having auditory neuropathy. Follow-up hearing tests were performed 6 months after the initial hearing tests, and the OAE and ABR tests showed the same re- sults, including robust OAE responses and no waveforms in the ABR test. His hearing thresholds had not changed but his speech discrimination scores improved to $44 \%$ in the right ear and $60 \%$ in the left ear. At present, he can easily communicate verbally with familiar people with aid of speech reading.

\section{Discussion}

When hearing loss is suspected, the highest priority must be to determine the type of hearing loss because this determines the diagnostic and therapeutic approaches used. Hearing loss is divided into two types: conductive hearing loss and sensorineural hearing loss. In the case of conductive hearing loss, the possibility of surgical management is considered

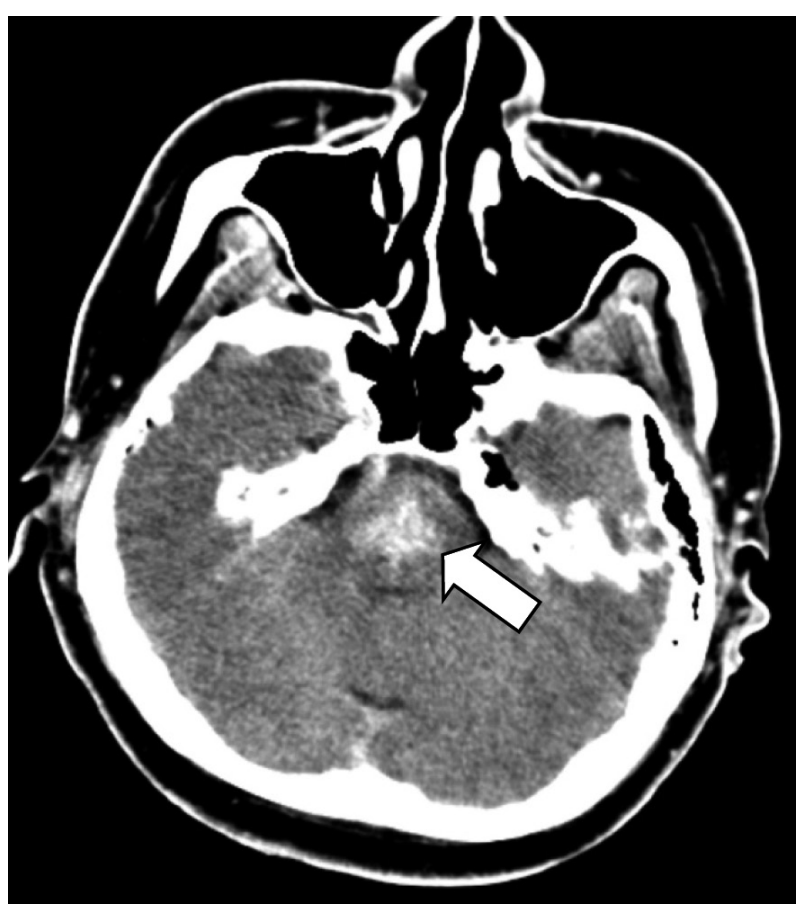

Fig. 1. The axial image of contrast-enhanced brain computed tomography demonstrates an acute hemorrhage in the central pons (white arrow).
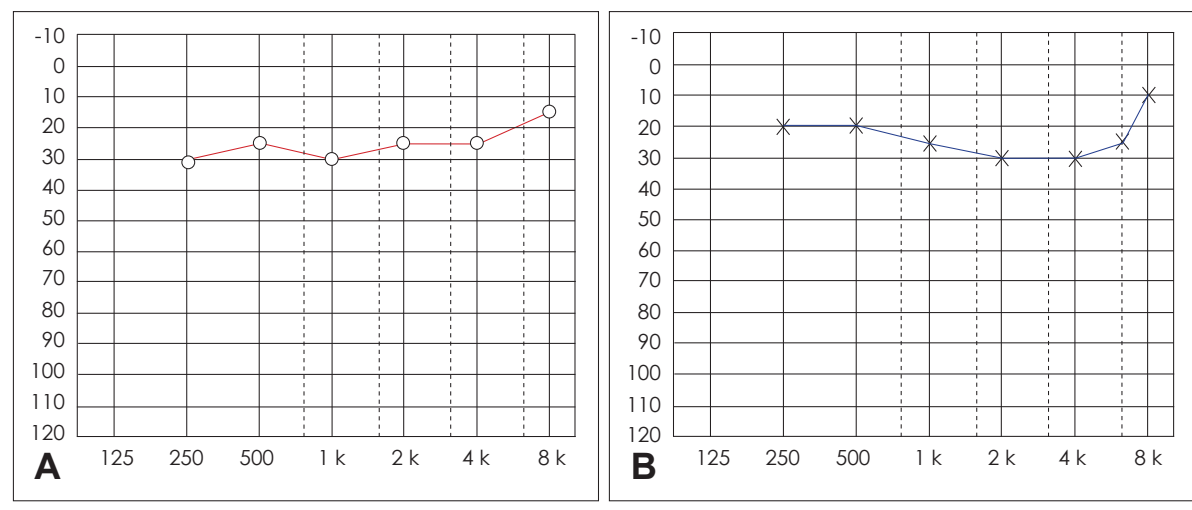

Fig. 2. Pure tone audiogram shows mild sensorineural hearing loss. A: Air conduction threshold of right ear. B: Air conduction threshold of left ear. 
first, but for sensorineural hearing loss, most cases cannot be cured surgically and hearing aids are the first choice. If the degree of hearing loss is severe, a cochlear implant can be con- sidered.

Sensorineural hearing loss accounts for about $90 \%$ of the total cases of hearing loss. Sensorineural hearing loss can oc-

Fig. 3. Transiently evoked otoacoustic emissions (OAE) (A) and distortion product OAE $(B)$ show robust responses in both ears.

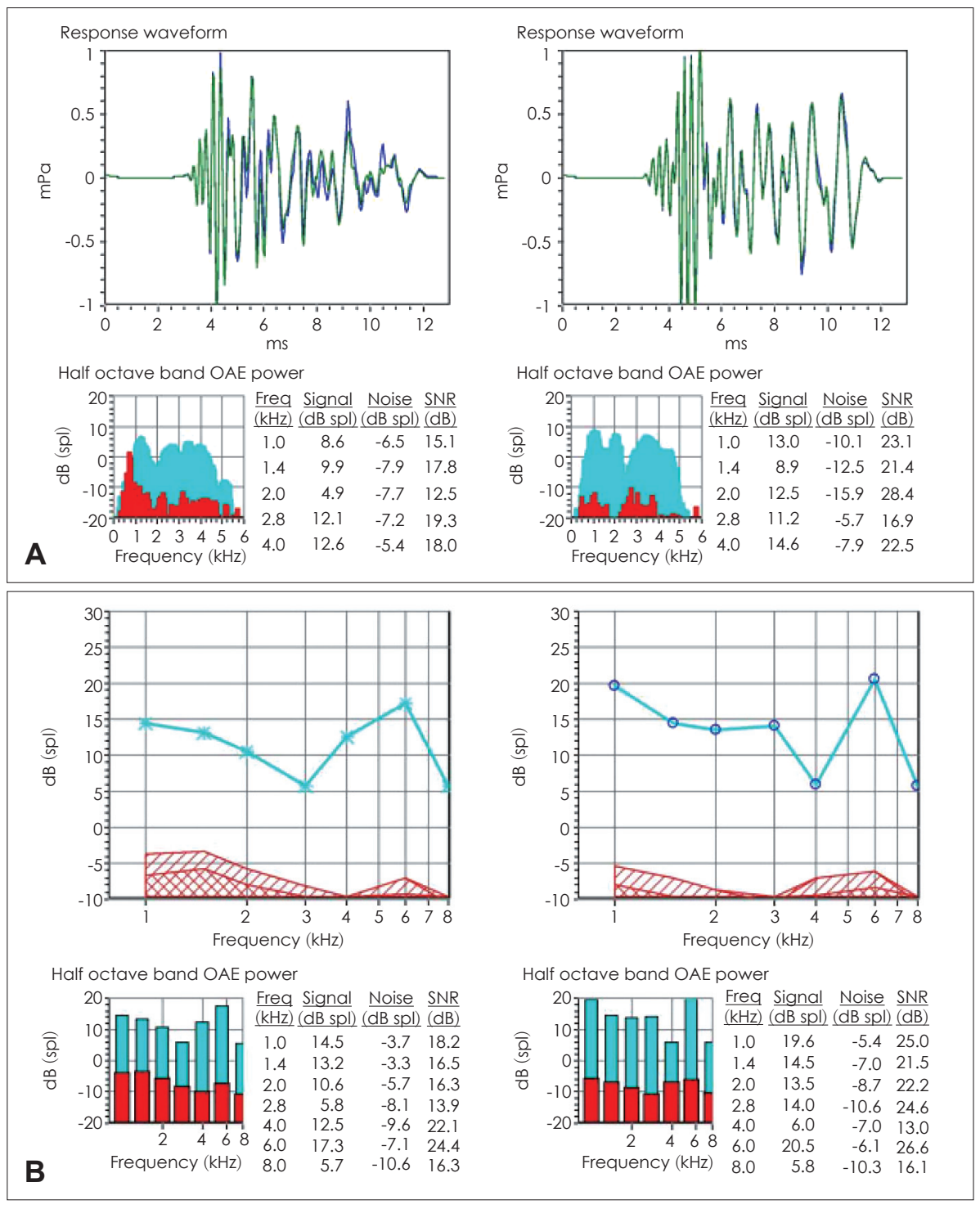

Fig. 4. Auditory brainstem responses show no discernible waveforms at stimulus using $90 \mathrm{~dB} \mathrm{nHL}$ click sounds. Only broad wave I (white arrow) was recorded at maximum stimulus level of $100 \mathrm{~dB} \mathrm{nHL}$.

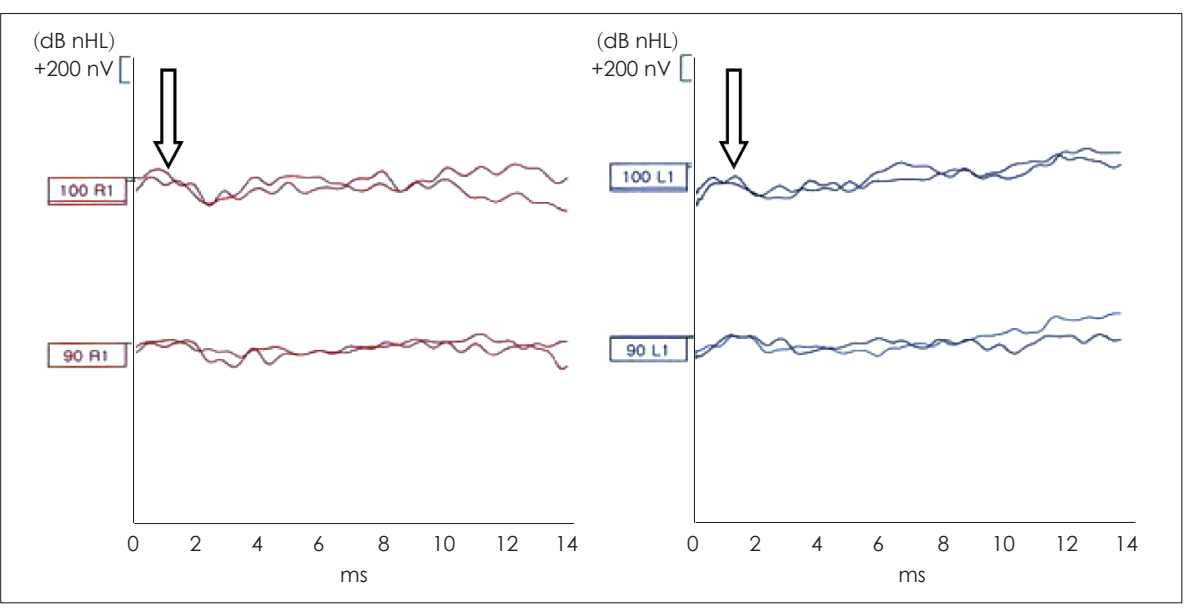


cur after damage to an auditory conduction pathway through the cochlea, auditory nerve, brainstem, or auditory cortex. In general, it is very rare that the origin of sensorineural hearing loss is damage to a central auditory conduction pathway. Clinically, most cases of sensorineural hearing loss are caused by pathology of the cochlea. The outer hair cells of the cochlea are the primary sites which are involved, but sometimes the inner hair cells are also affected. No response to an OAE test reflects dysfunction of the outer hair cells. The ABR threshold is also raised depending on the degree of damage to the hair cells. In some cases of sensorineural hearing loss, OAE responses are preserved but $\mathrm{ABR}$ responses appear absent or severely abnormal. In this case, an auditory neuropathy can be diagnosed [6]. Patients with an auditory neuropathy have quite normal function of the outer hair cells, but pathologies are in the inner hair cells or in an auditory conduction pathway from a type I auditory nerve to the brainstem [6,7].

In sensorineural hearing loss, the speech discrimination score is also decreased according to the elevation of the hearing threshold. Consequently, hearing aids can be applied in the case of slight or moderate hearing loss. If the hearing loss is severe or profound, cochlear implants can be applied to relieve extremely poor speech intelligibility. However, auditory neuropathy very commonly features poor speech discrimination regardless of the degree of hearing loss, in which case hearing aids do not assist speech intelligibility. In the cases of an auditory neuropathy caused by lesions in the inner hair cells, cochlear implants will be helpful, but their results will not be good if there are lesions in an auditory nerve or the brainstem. Thus, because of differences in the methods and effects of treatment, it is very important to identify an auditory neuropathy in patients with sensorineural hearing loss. If infants with suspected hearing loss attend the hospital, an auditory neuropathy can be identified without difficulty because impedance audiometry, OAE and ABR are routinely checked. However, because adults with suspected hearing loss preferentially undergo tests such as pure tone audiometry and a speech discrimination test, auditory neuropathy can often be missed. Because auditory neuropathy has a high prevalence of up to $10 \%$ of cases of total sensorineural hearing loss [8], if an auditory neuropathy is suspected clinically, it is necessary to perform additional tests of OAEs and ABRs to detect it.

Auditory neuropathy can result in a variable hearing threshold from normal to profound deficits, and typically results in a poor speech discrimination score [6,7]. Therefore, if a patient's speech discrimination score is decreased more than expected from the threshold level of a pure tone audiogram, it is advantageous to perform additional OAE and ABR tests to identify an auditory neuropathy. The age of onset of auditory neuropathy varies from birth to the $60 \mathrm{~s}$, but most cases are congenital [9]. Cases of an acquired auditory neuropathy have been reported to occur as hereditary progressive hearing loss and after viral infections $[10,11]$, but there is no previously reported case of an auditory neuropathy as a result of acute brain lesions such as brain infarction or hemorrhage; our case is the first report of acquired auditory neuropathy developed after brainstem hemorrhage.

Hearing loss after stroke mainly occurs because of infarction of the anterior-inferior cerebellar artery [12], and hearing loss after a pontine hemorrhage is very rare [13-15]. Auditory information is conducted via the cochlea, vestibulocochlear nerve, cochlear nucleus, lateral lemniscus, inferior colliculus, and auditory cortex. The cochlear nucleus is bilaterally connected to the inferior colliculus, so each auditory cortex receives auditory information passed from both sides of the cochlear nucleus. Therefore, bilateral hearing loss resulting from a pontine hemorrhage is estimated to be rare, but can occur when a massive hemorrhage occurs in the bilateral cochlear nuclei. In our patient, bilateral hearing loss occurred because of the large volume of bleeding (about $5 \mathrm{~mL}$ ) in the central pontine region that contains the bilateral cochlear nuclei.

The axonal or demyelinating lesion of the auditory nerve are known to be the major pathophysiology of auditory neuropathy, which results in the failure of precise synchronization of the action potential generated at each nerve fiber and cause abnormal ABR waveforms [16]. Pontine hemorrhage can affect the ABR waveform because a duration of acute nerve compression of over 2 hours can cause Wallerian degeneration of neural tissue; if more time passes, extensive neural degeneration results [17]. Waves III-V of the ABR were lost in animals with a damaged trapezoid body [18]. In older patients in whom pontine infarction occurred in the lower end of the brain stem, ABR waves II-V were reported to be missing [19]. Our patient did not show any waveforms from the ABR except wave I. We thought that the disappearance of the waveforms II-V was caused by a large central pontine hematoma that compressed the bilateral cochlear nuclei in the pons.

Varying degrees of recovery of hearing loss caused by pontine hemorrhage have been reported. In the case of a 64-yearold woman who had hearing loss caused by a pontine hemorrhage [14], the initial bilateral auditory threshold tests indicated mild hearing loss, while the speech discrimination scores on both sides were reduced to $0 \%$. The patient recovered normal hearing after 1 year. Goyal, et al. [15] reported that a 51-yearold patient with pontine hemorrhage showed a unilateral high-frequency hearing loss and a decrease in both speech discrimination scores ( $52 \%$ on the right side, $24 \%$ on the left side). This patient also recovered normal hearing thresholds 
and his speech discrimination scores recovered to $80 \%$ and $88 \%$ after 6 months. The patient of this report who had auditory neuropathy after pontine hemorrhage showed partial recovery. Although he complained of hearing loss, hearing aid was not prescribed because hearing thresholds for both ears were well preserved $(26 \mathrm{~dB}$ HL on the right ear and $32 \mathrm{~dB}$ $\mathrm{HL}$ on the left ear). Fortunately the speech discrimination improved over time from zero to $44 \%$ in the right ear and $60 \%$ in the left ear, so he can easily communicate verbally with familiar people with aid of speech reading. In this case, it is important to incorporate communication strategies such as looking at the patient when talking to him/her and minimizing the level of background noise.

\section{Conflicts of interest}

The authors have no financial conflicts of interest.

\section{REFERENCES}

1) Jang SH, Chang MC. Recovery of an injured corticoreticulospinal tract in a patient with pontine hemorrhage. Int J Stroke 2016;11:NP $18-9$.

2) Moncayo J. Pontine infarcts and hemorrhages. Front Neurol Neurosci 2012;30:162-5.

3) Park JS, Sun WS, Pyo SY, Kim MS, Jeong YG, Lee SI, et al. Clinical aspect of spontaneous pontine hemorrhage. Korean J Cerebrovasc Surg 2005; 7:31-6.

4) Takeuchi S, Suzuki G, Takasato Y, Masaoka H, Hayakawa T, Otani $\mathrm{N}$, et al. Prognostic factors in patients with primary brainstem hemorrhage. Clin Neurol Neurosurg 2013;115:732-5.

5) Muttikkal TJ, Kesser BW, Mukherjee S. Isolated contralateral sudden sensorineural hearing loss: an unusual manifestation of pontine infarct. Neuroradiol J 2014;27:483-6.

6) Starr A, Picton TW, Sininger Y, Hood LJ, Berlin CI. Auditory neu- ropathy. Brain 1996;119(Pt 3):741-53.

7) Rapin I, Gravel J. "Auditory neuropathy": physiologic and pathologic evidence calls for more diagnostic specificity. Int J Pediatr Otorhinolaryngol 2003;67:707-28.

8) Hood LJ. Auditory neuropathy/dys-synchrony disorder: diagnosis and management. Otolaryngol Clin North Am 2015;48:1027-40.

9) Rance G. Auditory neuropathy/dys-synchrony and its perceptual consequences. Trends Amplif 2005;9:1-43.

10) Manchaiah VK, Zhao F, Danesh AA, Duprey R. The genetic basis of auditory neuropathy spectrum disorder (ANSD). Int J Pediatr Otorhinolaryngol 2011;75:151-8.

11) Prabhu P. Acquired auditory neuropathy spectrum disorder after an attack of chikungunya: case study. Eur Arch Otorhinolaryngol 2016; 273:257-61.

12) Kim HA, Lee BC, Hong JH, Yeo CK, Yi HA, Lee H. Long-term prognosis for hearing recovery in stroke patients presenting vertigo and acute hearing loss. J Neurol Sci 2014;339:176-82.

13) Cohen M, Luxon L, Rudge P. Auditory deficits and hearing loss associated with focal brainstem haemorrhage. Scand Audiol 1996;25: 133-41.

14) Egan CA, Davies L, Halmagyi GM. Bilateral total deafness due to pontine haematoma. J Neurol Neurosurg Psychiatry 1996;61:628-31.

15) Goyal MK, Kumar G, Sahota PK. Reversible sensorineural hearing loss with normal brainstem auditory evoked potentials in pontine hemorrhage due to capillary telangiectasia. J Clin Neurosci 2010;17: 1198-201.

16) Rance G, Starr A. Pathophysiological mechanisms and functional hearing consequences of auditory neuropathy. Brain 2015;138(Pt 11): 3141-58.

17) Tseng TJ, Hsiao TH, Hsieh ST, Hsieh YL. Determinants of nerve conduction recovery after nerve injuries: compression duration and nerve fiber types. Muscle Nerve 2015;52:107-12.

18) Wada SI, Starr A. Generation of auditory brain stem responses (ABRs). II. Effects of surgical section of the trapezoid body on the ABR in guinea pigs and cat. Electroencephalogr Clin Neurophysiol 1983;56: $340-51$.

19) Deplanque D, Godefroy O, Guerouaou D, Laureau E, Desaulty A. Sudden bilateral deafness: lateral inferior pontine infarction. J Neurol Neurosurg Psychiatry 1998;64:817-8. 